\title{
7. Die Transformation von ländlichen Räumen und den darin lebenden Gesellschaften in Westdeutschland
}

\subsection{Einleitung}

Die Transformation ländlicher Räume im westlichen Europa ist eng verbunden mit der europäischen Integration - und dabei vor allem mit der Gemeinsamen Agrarpolitik - sowie dem demographischen und sozialen Wandel. Deren Richtlinien und Auswirkungen hat die Gesellschaft im ländlichen Raum stark geprägt. Dies hat bereits seit den frühen 1950er Jahren Auswirkungen auf die Entwicklungen in westdeutschen ländlichen Regionen, während in den ostdeutschen Regionen zunächst auf Grundlage der Kollektivierung und der sozialistischen Planwirtschaft andere Voraussetzungen existierten, auf die aber in diesem Kapitel nicht näher eingegangen werden soll.

Es stellt sich zunächst die Frage, was überhaupt „der ländliche Raum“ sein soll, da die Vielfalt der Räume, die unter diesem Begriff subsumiert werden, sehr groß ist. So gehören die touristisch attraktiven Regionen der Nord- und Ostseeküste oder des Bayerischen Waldes genauso dazu, wie die landwirtschaftlichen Intensivregionen Nordwestdeutschlands, die Weinregionen Frankens oder der Mosel oder die Regionen kleinbäuerlicher extensiver Landwirtschaft wie in der Lüneburger Heide oder auf den Almen. Die Europäische Union und die OECD nutzen die statistische Definition, nach der Regionen mit einer Einwohnerzahl kleiner als 150 Einwohner pro $\mathrm{km}^{2}$ als ländlich gelten. Auch wenn diese Definition wenig mit der Realität vieler ländlich geprägter Räume zu tun hat, bildet sie dennoch die Grundlage für die Verteilung von EU-Finanzmitteln sowie für regionale und nationale Statistiken der europäischen Staaten.

Dabei hat die Gemeinsame Agrarpolitik immer wieder zu Veränderungen der Landnutzung geführt, da die Land- und Forstwirtschaft für viele Jahrzehnte die dominanten Branchen in den ländlichen Räumen darstellten. Jedoch hat seit den 1990er Jahren ein Paradigmenwechsel hin zu einer Multifunktionalität ländlicher Räume mit neuen oder stärker in den Fokus gerückten Funktionen (z.B. Energiegewinnung, Naturschutz) neben der rein landwirtschaftlichen stattgefunden, was Auswirkungen auf die ländliche Gesellschaft dieser Räume hat. Ferner haben auch allgemeine demographische Entwicklungen oder die Anpassung ländlicher an städtische Sozialformen wichtige Auswirkungen seit dem zweiten Weltkrieg auf die ländlichen Räume Westdeutschlands gehabt, wie dieser Beitrag zeigen soll.

\subsection{Veränderungen der ländlichen Räume und ihrer Gesellschaft seit dem Zweiten Weltkrieg}

\subsubsection{Die Entwicklung ländlicher Räume durch die Gemeinsame Agrarpolitik (GAP) seit den 1950er Jahren}

Bereits durch die sogenannten Römischen Verträge (Art. 33 EG Vertrag), die die Rechtsgrundlage für die Europäische Wirtschaftsgemeinschaft 1956 legten, wurden die Ziele der Gemeinsamen Agrarpolitik festgelegt. Die Gemeinsame Agrarpolitik betraf zunächst die Gründerstaaten (West-)Deutschland, Frankreich, Italien, Belgien, Luxemburg und die Niederlande. Nach dem Zweiten Weltkrieg und den danach entstehenden Versorgungsengpässen stand zunächst die Produktivitätssteigerung durch die Förderung des technischen Fortschrittes zur Sicherstellung der Versorgung zu bezahlbaren 
Verbraucherpreisen im Vordergrund. Außerdem sollte den in der Landwirtschaft tätigen Personen eine angemessene Lebenshaltung ermöglicht werden (u. a. Buttimer und McGauran 1994, S. 5f.; Dannenberg und Chilla 2012, S. 268). Daher wurden gemeinsame Wettbewerbsregeln und eine gemeinsame Marktordnung festgelegt. 1962 wurde dann der Europäischer Ausrichtungs- und Garantie- Fonds für die Landwirtschaft (EAGFL) als Finanzinstrument eingerichtet, der neben der Preisstützung auch Strukturreformen in der Landwirtschaft und in den ländlichen Räumen der Mitgliedsstaaten anschieben und finanzieren sollte (u. a. Dannenberg und Chilla 2012, S. 268; Klohn und Voth 2010, S. 32f.). Da jedoch die Preisstützung den Hauptförderbereich darstellte, kam es in der Folge - vor allem in den 1980er Jahren - durch Überproduktion zur Entstehung der sogenannten Milchseen und Rinderberge sowie durch den hohen Einsatz an Produktionsmitteln zu Umweltproblemen (u. a. Europäische Kommission 2005). Als Gegenstrategie kamen die Einführung von Milchquoten oder Garantieschwellen in den 1980er Jahren. Bis zu diesem Zeitpunkt war der Strukturwandel in der Landwirtschaft auf eine deutliche monofunktionale Produktionssteigerung ausgerichtet. Darüber hinausgehende Aspekte der ländlichen Räume, die vor allem soziale Ausrichtung besaßen oder beispielsweise einer zukunftsorientierten Dorferneuerung dienten, wurden weitestgehend außer Acht gelassen.

Grundlegend für eine Hinwendung zu einem integrierten Ansatz, der ländliche Räume als mehr versteht als nur landwirtschaftlich strukturiert, war zunächst das Dokument „The Future of Rural Societies“ der Europäischen Kommission von 1988 und dann die „Erklärung von Cork 1996“ mit der Erklärung der Folgekonferenz von Salzburg 2003 (Europäische Gemeinschaften. Kommission 1988; Europäische Kommission 1996 und Europäische Kommission. Generaldirektion Landwirtschaft 2003/2004). Die Erklärung von Cork beinhaltet ein 10-Punkte-Programm mit der Priorisierung ländlicher Räume, ihrer integrierten Entwicklung und Diversifizierung (Europäische Kommission 1996). Diese Neuausrichtung sollte nachhaltige Verbindungen von Landwirtschaft und ländlicher Gesellschaft bedeuten. „The emphasis now shifted to rural diversification, to bottom-up rather than topdown approaches, to support for indigenous businesses to the encouragement of local initiative and enterprise and, where these are weak, to the provision of suitable training" (Lowe et al 1995).Dabei sollten neue Kooperationen zwischen landwirtschaftlichen und nichtlandwirtschaftlichen Akteuren in den ländlichen Räumen angeregt werden (vgl. Ploeg et al. 2000). Hiermit rückt auch - und das war neu - die nicht-landwirtschaftlich tätige Bevölkerung der ländlichen Räume stärker in den Fokus der Aufmerksamkeit und somit auch in die Förderkulisse der Europäischen Union.

Um eine möglichst breite Partizipation, d.h. die Teilnahme und Teilhabe möglichst vieler Akteure im ländlichen Raum, und somit neue Zusammenarbeit und Verbindungen der Akteure in den ländlichen Räumen sowie eine breite Akzeptanz der neuen Projekte und Veränderungen zu erreichen, wurde die EU- Gemeinschaftsinitiative LEADER (=Liaison entre actions de développement de l'économie rurale) (1994-2006) initiiert. Die Vernetzung von Akteuren, Bottom-up-Ansatz, territorialer Ansatz und regionale Entwicklungskonzepte standen dabei im Mittelpunkt. Mit der Förderperiode 2007-2013 wurde LEADER dann zum sogenannten Mainstream-Förderinstrument als 4. Achse des neu geschaffenen Europäischen Landwirtschaftsfonds für die Entwicklung des ländlichen Raumes (ELER). Auch in der Landwirtschaft folgten weitere Reformen: MacSharry-Reform (1992), Agenda 2000 (1999) und der sog. „Health Check“ (2008) (u. a. Dannenberg und Chilla 2012, S. 268, Klohn und Voth 2010, S. 34f.), die sich aber vor allem mit landwirtschaftliche Produktionsformen auseinandersetzten und somit nur indirekt Auswirkungen auf die ländliche Gesellschaft haben. 


\subsubsection{Demographischer und sozialer Wandel}

Wie gravierend die demographischen und sozialen Transformationen der ländlichen Regionen Westdeutschlands und zum Großteil auch in anderen ländlichen Regionen Westeuropas sind, wird zunehmend untersucht.

Henkel (2004, S. 98) fasst den sozialen Wandel in ländlichen Räumen zusammen als „Lösung des Dorflebens von der Land- und Forstwirtschaft, Annäherung der Gegensätze und

Eigenarten zwischen Stadt und Land“. Hainz (1999; aus: Franzen et al 2008, S. 11f.) sieht den Wandel ländlicher Gesellschaften als genauso fundamental an, wie den städtischer, mit einer starken Ausdifferenzierung der Sozialstruktur und Lebensformen. Dieser Wandel beginnt bereits direkt nach dem Zweiten Weltkrieg

In dieser Zeit ist die demographische Entwicklung in den ländlichen Räumen Westdeutschlands zunächst durch Flüchtlinge, Vertriebene und Evakuierte gekennzeichnet. Die sogenannte Dorfstudie, eine Langzeitstudie die seit1952 in regelmäßigen Abständen die immer wieder gleichen Dörfer auf ihre Veränderungen hin untersucht, zeigt Dörfer im Jahr 1952 auf, in denen diese neu hinzugekommenen Personengruppen 40\% der Gesamtbevölkerung ausmachen, wobei die einzelnen Dörfer sehr unterschiedlich besiedelt werden (Dietze et al. 1953, S. 103). Gefolgt wurde diese Zuwanderung dann in den 1950er Jahren von einer Abwanderung und damit von Bevölkerungsschrumpfungen im ländlichen Raum, da eine soziale Integration der Flüchtlinge, Vertriebene und Evakuierte vielerorts nicht stattgefunden hatte (Becker 1997, S. 44). Ferner kam es in dieser Zeit zum Phänomen der sogenannten Sozialbrache. Landwirtschaftliche Nutzfläche fiel brach, da die Besitzer nichtlandwirtschaftliche Arbeitsstellen annahmen (Henkel 2004, S. 139). Dies hatte sowohl Auswirkungen auf die Kulturlandschaft als auch auf die Sozialstruktur, die durch eine Abnahme in der Landwirtschaft Beschäftigter gekennzeichnet ist. In den nachfolgenden Jahren verändern sich die Bevölkerungszahlen und -strukturen ländlicher Räume in der Nähe von Städten durch Suburbanisierungsprozesse, während peripher gelegene Räume weiterhin an Bevölkerung verloren (Deenen o.J., S. 66 f.). Becker (1997, S. 47ff.) sieht eine wichtige Umbruchphase zwischen 1987 und 1994. In dieser Phase wachsen erneut nicht nur die stadtnahen, sondern auch die peripher gelegenen ländlichen Regionen in Westdeutschland bedingt durch die Ausweisung von Baugebieten, womit die Dörfer zunehmend zu Wohngebieten abgekoppelt von potenziellen Erwerbsmöglichkeiten werden. Außerdem nehmen die Fluktuation und die Mietwohnverhältnisse der Einwohner zu und verändert somit das bisherige Sozialgefüge, welches häufig durch Persistenz und Eigentum gekennzeichnet waren.

Becker (1997, S. 52 ff.) hebt als neu hinzugezogene Personengruppen, die diese bisherigen Sozialgefüge verändern, junge gut gebildete Familien, Rentner, Spätaussiedler und in einem geringen Maße auch internationale Migranten hervor. Durch diese Neubürger der Dörfer verändert sich ferner auch die ökonomische Basis, so dass neben dem durch die GAP unterstützten Agrarstrukturwandel auch der Zuzug dieser Personengruppen zu einer Abnahme der Bedeutung der Landwirtschaft als ökonomische Grundlage der westdeutschen Dörfer geführt hat. Dies wiederum verstärkte die soziale Transformation. „Allerdings bleiben bei Bauern im Bereich von Alltagskultur und Freizeitverhalten traditionelle Muster deutlich sichtbar, denn die soziale Gruppenzugehörigkeit, die naturnahen Arbeitsbedingungen, die "Unentrinnbarkeit" von Haus und Hof schnüren einen Alltag, der wenige Variationsspielräume zulässt. Neben den traditionellen Alteingessenen wohnen in Dörfern zunehmend Personen mit hohen Freizeitansprüchen und einem Lebensstil, der als "ländliche Moderne" bezeichnet werden kann (Garten-Park, Sportausrüstung, etc.). Weitere Gruppen bilden Personen mit kritischer oder avantgardistischer Haltung, die z.B. Bürgerinitiativen bilden, neue Arbeitsplätze schaffen und Kneipen und Direktvermarktung betreiben und solche, 
die ausgegrenzt sind oder sich selbst isolieren, z.B. in spezifischen (auch religiös geprägten) Milieu-Treffpunkten.“ (Spellerberg 2012, S. 408)

Klohn und Voth (2010, S. 21) stellen fest, dass sich durch den Bedeutungsrückgang der Landwirtschaft auch deren politische Einflussnahme in den Dörfern verringert hat und somit zunehmend auch Proteste durch nicht-landwirtschaftliche Dorfbewohner gegen

landwirtschaftliche Projekte wie beispielsweise den Bau von Ställen oder Massentierhaltung entstehen. Ferner zieht es zunehmend alternative Lebensstilgruppen in ländliche Räume, um dort beispielsweise nach christlich-ökologischen, ökologischen oder anderen Prinzipien zu leben (s. hierzu u. a. Woods 2005, S. $291 \mathrm{ff}$.).

Ferner sind viele, vor allem peripher gelegene Räume neben den Veränderungen der Sozialformen und -strukturen durch eine Überalterung der Bevölkerung gekennzeichnet. Aber auch hier muss, wie für alle Faktoren, ein differenziertes Bild dargelegt werden. So gibt es beispielsweise mit den Landkreisen Cloppenburg und Vechta in Niedersachsen Regionen mit hohem natürlichen Bevölkerungswachstum und einer dementsprechend vergleichsweise niedrigen Altersstruktur (Niedersächsischer Landtag 2007, S. 66ff.), während auf der anderen Seite Regionen wie die Westeifel durch besonders hohe Überalterung, eine geringe Fertilitätsraten und hohe Abwanderungsraten junger, zumeist gut ausgebildeter Personen gekennzeichnet sind. Gerade in ländlich peripheren Räumen sind Schrumpfungs- und Alterungsprozesse (nicht nur) in Westdeutschland vorzufinden (Reichert-Schick 2010, S. 154).Die Überalterung zeigt sich beispielsweise in den ländlich geprägten Landkreisen Harz, Goslar und Lüchow-Dannenberg in Niedersachsen durch einen Anteil an 60-Jährigen und Älteren an einer Gesamtbevölkerung von über 30\% (2006). Der Anteil der 80-Jährigen und Älteren macht im Landkreis Goslar sogar knapp 7\% (2006) der Gesamtbevölkerung aus(Niedersächsischer Landtag 2007, S. 72ff.). Durch die immer stärker alternde Gesellschaft sind die ländlichen Regionen vor besondere Herausforderungen gestellt. So entstehen unter anderem mobile Pflegeeinrichtungen oder Mehrgenerationenhäuser in ländlichen Regionen, um diesen neuen Anforderungen zu entsprechen. Auch zeigt sich, dass immer mehr Vereine, z.B. auch die Freiwillige Feuerwehr, auf Grund des Nachwuchsmangels mit den Vereinen anderer Dörfer zusammengelegt werden müssen und somit zum einen Vereinsleben vor Ort, zum anderen aber auch die Sicherung gewisser Infrastrukturdienstleistungen durch die Vereine verloren geht (z.B. ehrenamtlicher Hochwasserschutz oder ehrenamtliche „TanteEmma“"Läden). Damit verschwinden Teile des Sozialkapitals - als wechselseitige Beziehungen, Netzwerke und Werte verstanden - ländlicher Räume, die bisher das dörfliche Leben in vielen Orten prägten.

Der demographische Wandel westdeutscher ländlicher Räume bezieht sich neben der Abnahme der Bevölkerungszahlen und der Überalterung der Bevölkerung auch auf eine Internationalisierung der Bevölkerung durch internationale Migranten. Dieses Phänomen ist für Städte sehr gut untersucht, aber auch in einigen ländlichen Räumen kann man eine deutliche Zunahme der Migrantenzahlen feststellen. Dies hat verschiedene Ursachen. Zum einen wurden die sogenannten Spätaussiedler in den 1990er Jahren aus den Gebieten der ehemaligen Sowjetunion von der Außenstelle Friedland des Bundesverwaltungsamtes auf alle Gemeinden innerhalb Deutschlands verteilt, so dass hier auch ländliche Orte einen Zuzug erfuhren (Bundesverwaltungsamt o.J.). Zum anderen erfolgt ein freiwilliger Zuzug von Migranten auf Grund von Erwerbsmöglichkeiten oder auf Grund der günstigen Immobilienpreise. Die günstigen Immobilienpreise spielen gerade im Grenzraum eine bedeutende Rolle. So steigt seit ca. 2000 vor allem die Zahl der Niederländer oder Luxemburger auf der deutschen Seite der jeweiligen Grenze. Hierbei spielen die steigenden Immobilienpreise und eine boomende Wirtschaft auf der anderen Grenzseite eine entscheidende Rolle(z. B. Houtum und Gielis 2006; Nadler et al 2012; Nienaber und Kriszan [angenommen]). Grenzgemeinden, wie die saarländische Gemeinde Perl, erreichen so beispielsweise Anteile ausländischer Mitbewohner von 20,7\% bzw. einen Anteil von 
Luxemburgern von 14,0\% im Jahr 2008 (Auskunft der Gemeinde Perl 2012).Problematisch hierbei kann ein fehlender Integrationswille seitens der Zugezogenen, aber auch der Einheimischen sein, da der Lebensalltag weiterhin auf der anderen Seite der Grenze stattfindet und sich in Neubaugebieten luxemburgische Enklaven bilden (Nienaber und Kriszan[angenommen]). Ferner gibt es im ländlichen Raum noch die sogenannten ,amenity“Migranten („Wohlfühlmigranten“), die sich durch die Landschaft, Ruhe und andere „Wohlfühl"-Faktoren in den ländlichen Raum gezogen fühlen. Diese Migranten können sowohl internationale als auch Binnenmigranten sein. Bei den nationalen Binnenmigranten handelt es sich häufig zusätzlich um sogenannte Ruhesitzmigranten, die nach dem Eintritt in die Rente in diese Räume ziehen. Sie verstärken damit jedoch das Phänomen und die damit verbundenen Auswirkungen der Überalterung dieser Räume. Die ,amenity“-Migration ist vor allem in landschaftlich und/oder touristisch attraktiven Gebieten zu finden (u.a. Glorioso 2009) und verändert dort ebenfalls die ländliche Gesellschaft, indem neue Sozialstrukturen und Bevölkerungsgruppen in diese Orte ziehen.

\subsection{Fazit}

Die verschiedenen dargestellten Faktoren der Transformation ländlicher westdeutscher Räume zeigt die Vielfalt der Einflüsse auf, die im Rahmen eines solchen Kapitels weder in Gänze, noch differenziert nach Regionen dargelegt werden können. Daher sollen die genannten Regionen nur als Beispiele der Entwicklungen stehen.

Zusammenfassend sind die Entwicklungen durch die Gemeinsame Agrarpolitik — weg von stark monostrukturierten Räumen hin zu multifunktionalen, diversifizierten Räumen — , die Veränderung der Sozialstruktur mit einer immer stärkeren Anpassung an urbane Lebensformen sowie die Veränderung der Bevölkerungsstruktur im Rahmen des demographischen Wandels hier zu nennen. Besonders die letzt genannten Transformationen haben Auswirkungen auf den Erhalt des dörflichen Gemeinschaftslebens und der örtlichen Infrastrukturen (z.B. im Bereich des Gesundheitswesens, des Bildungswesens, des Öffentlichen Personennahverkehrs, des Vereinswesens oder auch des Einzelhandels), so dass zukünftig verstärkt neue Formen des dörflichen Zusammenlebens und der Infrastruktursicherung (z.B. mobile Einzelhandelsversorgung) oder auch der Zusammenlegung von Infrastrukturen über einzelne ländliche Gemeinden hinaus Aufgabe der Gesellschaft sein werden.

Insgesamt ist davon auszugehen, dass sich die hier dargelegten Transformationen in den kommenden Jahren noch verstärken und somit die Herausforderung für die Einwohner der ländlichen Räume Westdeutschlands zunehmen werden.

\subsection{Literatur}

Becker, H. (1997). Dörfer heute. Ländliche Lebensverhältnisse im Wandel 1952, 1972 und 1993/1995. Bonn: Schriftenreihe der Forschungsgesellschaft für Agrarpolitik und Agrarsoziologie.

Bundesverwaltungsamt (Hrsg.) (o.J.). Spätaussiedleraufnahmeverfahren. http://www.bva.bund.de/cln_101/nn_372236/DE/Aufgaben/Abt_III/Spaetaussiedler/spaeta ussiedler-node.html? _nnn=true. Zugegriffen: 22. März 2013.

Buttimer, A. \& McGauran, A.M. (1994).The impact of the EC Agricultural Policies on the Irish Rural Landscape. Acta Universitatis Carolinae Geographica 1, 5-17.

Dannenberg, P. und Chilla, T.(2012). Zwischen Markt- und Planwirtschaft -Agrarwirtschaft nach der Systemkonkurrenz. In H. Gebhardt, R. Glaser\&S. Lentz (Hrsg.).Europa- eine Geographie (S. 262-271). Berlin/Heidelberg: Springer. 
Deenen, B.v. (o.J.). Grunddaten der Entwicklung in den Untersuchungsdörfern. In B. v. Deenen, H. J. Herlemann, M. Köhne, E. Mrohs, U. Planck, \& H. Spitzer (Hrsg.). Lebensverhältnisse in kleinbäuerlichen Dörfern 1952 und 1972. (S. 49-124). Bonn: Forschungsgesellschaft für Agrarpolitik und Agrarsoziologie.

Dietze, C.V., Rolfes, M. \&Weippert, G. (1953). Lebensverhältnissen in kleinbäuerlichen Dörfern. Berichte über Landwirtschaft. Sonderheft 157.

Europäische Gemeinschaften. Kommission (1988). Die Zukunft des ländlichen Raums. Mitteilungen der Kommission, dem Rat und dem Europäischen Parlament am 29. Juli 1988 vorgelegt (KOM (88) 501 endg). Luxemburg.

Europäische Kommission (1996). Erklärung von Cork - Ein dynamischer ländlicher Raum. http://ec.europa.eu/agriculture/rur/cork_de.htm. Zugeggriffen: 15. März 2013.

Europäische Kommission. Generaldirektion Landwirtschaft (2003/2004). Europäische Konferenz über ländliche Entwicklung in Salzburg. Newsletter 60.

Europäische Kommission (2005). GAP - Die Gemeinsame Agrarpolitik erklärt. http://www.eu-info.de/static/common/files/1834/GAP\%20\%20Die\%20Gemeinsame\%20Agrarpolitik\%20erklrt.pdf. Zugegriffen: 22.März 2013.

Franzen, N., Hahne, U., Hartz, A., Kühne,O., Schafranski, F., Spellerberg, A. und Zeck, H. (2008). Herausforderung Vielfalt - LändlicheRäume im Struktur- und Politikwandel. ARLE-Paper 4. http://shop.arl-net.de/media/direct/pdf/e-paper_der_arl_nr4.pdf. Zugegriffen: 22 . März 2013.

Glorioso, R. S. (2009). Managing Amenity Migration: The Role of Multiple Future Scenarios.Die Erde 140/3, 293-315.

Hainz, M. (1999).Dörfliches Sozialleben im Spannungsfeld der Individualisierung. Bonn: Forschungsgesellschaft für Agrarpolitik und Agrarsoziologie e.V.

Henkel, G. (2004). Der Ländliche Raum. Gegenwart und Wandlungsprozesse seit dem 19. Jahrhundert in Deutschland. Berlin/Stuttgart: Gebrüder Borntraeger Verlagsbuchhandlung.

Houtum, H. van \& Gielis, R. (2006). Elastic migration: the case of Dutch short-distance transmigrants in Belgian and German borderlands. Tijdschrift voor Economische en Sociale Geografie 97, 2, 195-202.

Klohn, W. \& Voth, A. (2010). Agrargeographie. Darmstadt: Wissenschaftliche Buchgesellschaft.

Lowe,P., Murdoch, J. \& Ward, N. (1995).Networks in Rural Development: Beyond Exogenous and Endogenous Models. In J.D. Ploeg\&G. Dijk(Hrsg.),Beyond Modernization. The Impact of Endogenous Rural Development (S. 87-105). Assen: Van Gorcum.

Nadler, R., Kriszan, M., Nienaber, B. \& Frys, W. (2012).Zuwanderung internationaler Migranten in schrumpfende ländliche Regionen: die Fallbeispiele Ostsachsen und Saarland. Europa regional 18/2010 (2012) 2/3, 107-121.

Niedersächsischer Landtag (2007). Bericht der Enquete-Kommission "Demographischer Wandel - Herausforderung an ein zukunftsfähiges Niedersachsen ". Hannover.

Nienaber, B. \& Kriszan, A. (angenommen). Entgrenzte Lebenswelten: Wohnmigration als Ausdruck transnationaler Lebensentwürfe im deutsch-luxemburgischen und deutschpolnischen Grenzraum. Raumforschung und Raumordnung

Ploeg, J. D. et al (2000). Rural Development: From Practices and Policies towards Theory. SociologiaRuralis 40 (4), S. 391-408.

Reichert-Schick, A. (2010). Auswirkungen des demographischen Wandels in regionaler Differenzierung: Gemeinsamkeiten und Gegensätze ländlich peripherer Entleerungsregionen in Deutschland - die Beispiele Vorpommern und Westeifel. Raumforschung und Raumordnung. doi: 10.1007/s13147-010-0027-5

Spellerberg, A. (2012). Lebensstile in ländlichen Räumen Europas. In H. Gebhardt, R. Glaser \& S. Lentz (Hrsg.). Europa- eine Geographie (S. 408-409). Berlin/Heidelberg: Springer. Woods, M. (2005).Rural Geography. London et al: Sage Publications. 
